CLAWAR 2018: 21st International Conference on Climbing and Walking Robots and the Support Technologies for Mobile Machines, Panama City, Panama, 10-12 September 2018

\title{
INDOOR LOCALIZATION OF MOBILE ROBOTS WITH WIRELESS SENSOR NETWORK BASED ON ULTRA WIDEBAND USING EXPERIMENTAL MEASUREMENTS OF TIME DIFFERENCE OF ARRIVAL
}

\author{
OUSMANE ABDOULAYE OUMAR \\ School of Engineering, London South Bank University, 103 Borough Road, London, SE1 \\ $O A A, U K$ \\ TARIQ P. SATTAR \\ School of Engineering, London South Bank University, 103 Borough Road, London, SE1 \\ $O A A, U K$ \\ MOHAMMAD OSMAN TOKHI \\ School of Engineering, London South Bank University, 103 Borough Road, London, SE1
} $O A A, U K$

\begin{abstract}
This paper presents investigations into wireless localization techniques for mobile robots operat-ing in indoor environments. Localization systems can guide robots to perform different tasks such as monitoring children or elderly people, aid mobility of the visually impaired and localize mobile objects or packages in warehouses. They are essential for localization of robots operating in re-mote places that are inaccessible or hazardous to humans. Currently, ultra wide band (UWB) in indoor environments provides an accuracy of $24 \mathrm{~mm}$ under line of sight (LOS) or non-line of sight (NLOS) conditions in a working range of $160 \mathrm{~m}$ indoors. The work presented in this paper carries out experimental validation of localization algorithms using mobile robots and UWB signals. These are measured in LOS and NLOS environments. The measurements are performed with the UWB radio PulsON 410 (P410) and mobile robots (AmigoBot) with maximum travel-ling speed of $1 \mathrm{~m} / \mathrm{s}$ and equipped with an on-board computer, sonar, odometer, camera and inertial navigation system. Experimental results obtained for the system show positioning errors of less than $55 \mathrm{~mm}$.
\end{abstract}

\section{Introduction}

In recent years, ultra wide band (UWB) technologies have drawn significant interest in the wireless community [1]. The UWB is a generic term used to identify a radio technique that has been tested under different names since the midtwentieth century such as impulse radio, carrier-free radio, baseband radio, time domain radio, non-sinusoid radio and wide bandwidth on the radio. It was not 
until 1990 that the U.S. Department of Defense introduced the term UWB. UWB localization is superior in terms of accuracy and power consumption compared with general positioning system (GPS) and wireless local area network (WLAN) localization. It is thus more suitable for indoor location-based applications [2].

Currently, the great flexibility of UWB technology (variable bit rates, multiple modulations, low power consumption, low cost, etc) provides the opportunity for the emergence of new types of network that are pervasive and reconfigurable. Typically, sensor networks and distributed networks that require no infrastructure or limited infrastructure could eventually be made up of a large number of low cost UWB devices that understand an environment, that can adapt to it and operate autonomously. New requirements for localization have emerged in recent years. These requirements, which relate mainly to indoor environments (inside buildings), require precision and a quality of unmatched service (availability, reliability, sustainability, independence, continuity, etc). Many applications and services have emerged whose effectiveness depends mainly on localization performance. Representative examples include localization and monitoring of goods and people in buildings and / or areas at risk, institutional or opportunist looking for victims on the scene of disasters (fires, earthquakes, avalanches, etc), identification, operational monitoring of military urban guerrilla, supervision of prisoners etc., applications in industry telecommunications transport, and the medical sector.

A position location technique involves two steps: the measurement of relative distances (ranging) and positioning based on the measured ranges. The received signal strength (RSS), time difference of arrival (TDOA), angle of arrival (AOA) and time of arrival (TOA) are the four commonly used ranging techniques. Among these RSS and TDOA are of more interest with UWB.

Earlier studies have indicated that TDOA and RSS techniques are the best choices for indoor and outdoor localization. RSS localization techniques have been widely studied as an inexpensive solution for wireless indoor positioning systems in recent years. The RSS technique is, however, not always reliable, because the estimate of the distance depends on several factors such as the absence of a direct signal path, fading due to multipath, the channel characteristics and the distance between reference points. The TDOA technique is suitable for indoor and outdoor systems due to its high accuracy and low computational complexity.

To achieve the objective, the accuracy of indoor localization with UWB using TDOA experimental measurements was analysed with experiments using AmigoBot mobile robots and Time Domain PulsOn 410 radio transmitter/receivers. The experiments were conducted using UWB signals measured in line of sight (LOS) and non-line of sight (NLOS) environments and 
for location estimation the TDOA algorithm was used. The measurements were made with the UWB radio PulsON 410 systems tracking mobile robots (AmigoBot's) that were equipped with an on-board computer, sonar, odometer, encoder, camera and an inertial navigation system. These tests were done in an indoor environment with the AmigoBot robot programmed for different motion trajectories e.g. in a straight line, curved line, rotation, and return to the starting point (the coordinates of the reference point $(0,0)$ ). The tests were repeated several times and UWB localization compared with measurements made by the AmigoBot robot's own localization system.

The experimental tests conducted many times showed that the UWB accuracy is very good with the positioning error between 24 and $55 \mathrm{~mm}$ with the error never exceeding $55 \mathrm{~mm}$ with a working range of $160 \mathrm{~m}$. The difficulty of obtaining a very accurate positioning system is due to NLOS propagation conditions. The localization error increased with longer robot travel distance. Results indicate that a system that uses UWB gives much more accurate position measurement in indoor environments than other localization techniques (which are mostly applied in outdoor environments). UWB TDOA measurement should be used to locate devices because of low cost, low power consumption and little computational complexity and precise positioning capability. Hence, UWB TDOA systems are suitable for applications such as monitoring children or elderly people, visitor management, enforcing restricted zones, security and asset tracking.

\section{Materials}

The TDOA algorithm is validated by using UWB signals measured in LOS and NLOS. These are measured by using the PulsON 410 system, which is formed by tags (transmitters / receivers) supplied by the Time Domain corporation. The localization techniques used by the Amigo robots (AmigoBot) are used to compare the position measurement with UWB for accuracy.

The AmigoBot contains all of the basic components for autonomous sensing and navigation in a real-world environment, including battery power, drive motors and wheels, position/speed encoders, sonar range-finding sensors, and integrated accessories, all managed via an on-board micro controller and mobile-robot server software. In this study, UWB sensors are used to find and track multiple moving robots in an indoor environment. Four P410 sensors are employed each fitted with a UWB transmitter and a UWB receiver. The sensors use different code channels to prevent interference and to reduce the effects of multipath signals at the receiver. An environment scanning phase of thirty minutes duration is employed as a reference to determine signals reflected from non-stationary targets. UWB 
pulses are transmitted from the sensors at every millisecond by the transmitter and all reflected signals picked up by the receiver. UWB signals are characterized by the transmission of a few nanosecond duration pulses [3, 4, 5]. They have very high time resolution and localization precision, which make UWB sensors an ideal equipment for short-range radar sensor network applications [6, 7]. In this study, UWB sensors are employed for detecting and tracking multiple moving objects in an indoor environment in the context of passive localization [8,9].

In the experiments, there are four sensors (fixed nodes), which are constantly transmitting signals, and the reflected signals from a robot moving in an indoor environment are collected by each of these sensors and four different experiments are performed.

TDOA needs to have a direct path, time synchronization is required between pairs of base stations and this needs high temporal resolution at the receiver and the technique is accurate.

The frequency band allocated by the FCC to UWB ranges from $3.1 \mathrm{GHz}$ to $10.6 \mathrm{GHz}$ in single band or multiband, except for the strip of 5 to $6 \mathrm{GHz}$, which is the UNI band (Figure 2) and is reserved for industrial testing, scientific and medical applications.

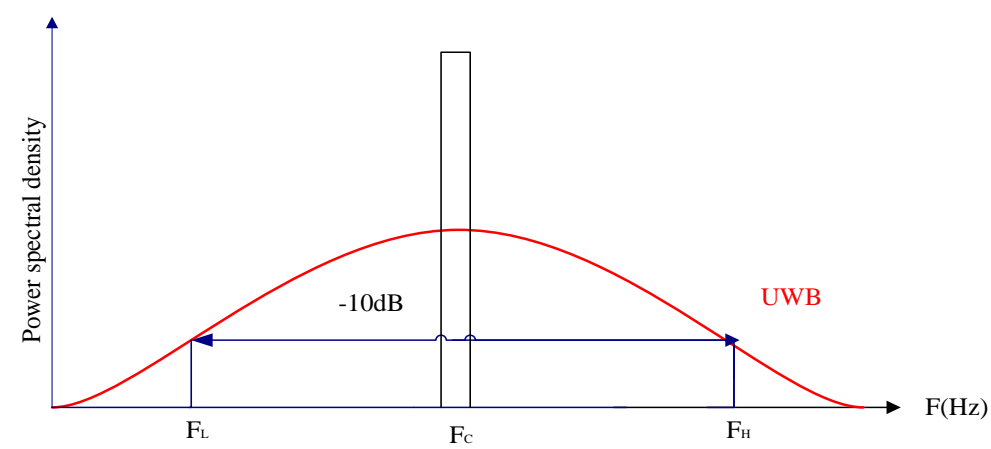

Figure 1: Illustration of the spectrum of UWB signal versus narrowband signal. 


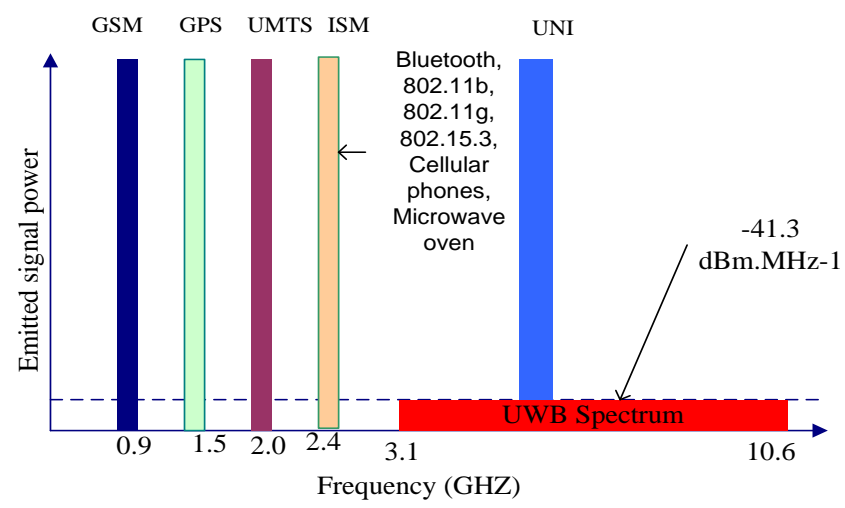

Figure 2: The different systems in ultra-high and super high frequency band.

The PulsON 410 transmitters and receivers equipment used for the experiment is the time domain P410 module, which is a small, low power UWB sensor device, and provides a very good accuracy, high rate range measurements and superior operational performance when compared to conventional radio frequency identification (RFID) and real-time locating systems (RTLS). P410 system consists of tags (transmitters / receivers) of the American firm Time Domain as shown in Figure 3.

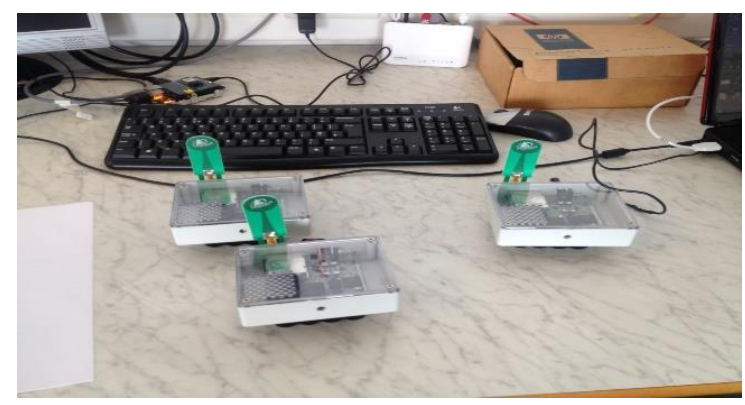

Figure 3: Three PulsOn 410 UWB sensors (each PulsOn 410 is in an enclosure).

The AmigoBots shown in Figure 4 are small robots developed by Adept Mobile Robots for research and education purposes. Each AmigoBot has a twowheel differential drive system with a rear-stabilizing wheel. It senses the environment through an array of eight sonar sensors [10]: six at the front and two at the rear, all of which detect the robot's proximity to its environment. The sonar sensors detect objects in their path at distances between approximately $15 \mathrm{~mm}$ to 
6 metres. Extremely close objects, less than $15 \mathrm{~mm}$, are seen by the sensors as being equivalent to over 6 metres away. An omnidirectional vision system allows the robot a 360-degree field of vision [11]. The sonar-firing rate of AmigoBot sensors is $25 \mathrm{~Hz}$ with distance measurement data sent wirelessly to other devices. The AmigoBot is also fitted with an internal coordinate system. It has two encoders, one fitted to each motor shaft and these are used as odometer to estimate the robot's position and orientation relative to its starting position. This gives a precise localization in a mapped space using robot odometry combined with laser rangefinder data implemented by the Advanced Robotics Navigation and Localisation (ARNL) laser localization library, and navigation software program along with mapping software tools and a laser rangefinder.

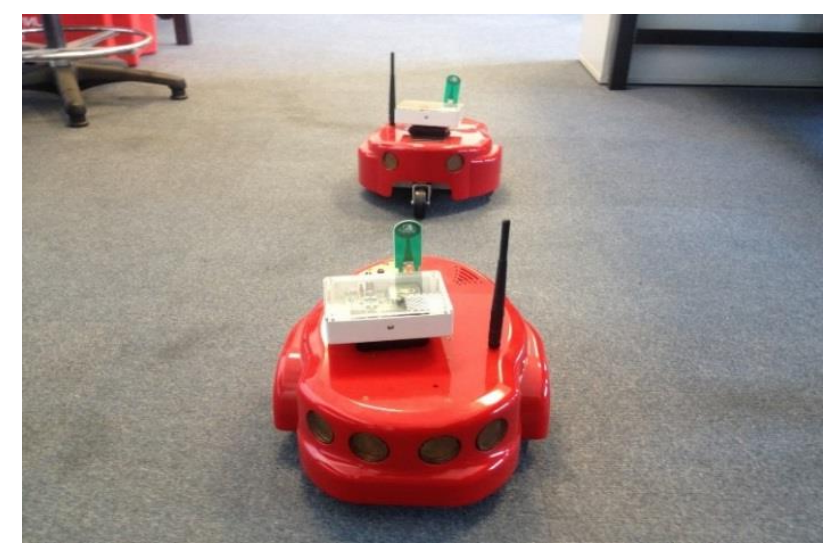

Figure 4: Two Amigo robots mounted with P410 in a testing environment.

\section{Estimation of TDOA and measurement environment}

To estimate TDOA with UWB, the signal is measured in both LOS and NLOS environments. Four antennas are connected to each channel of a laptop using wireless communication. The method of measuring the TDOA is equivalent to the measurement of TOA but without requiring knowledge of the initial time $t_{0}$. The exact position of each node is known. Figure 5 shows the measurement system used in the experiments. The measurement system displays the time stamp (consecutive measurements updated every second), range and velocity information. Nodes 100, 104, 105 and 106 are fixed nodes while 107 is a mobile node and node 100 is regarded as node of reference. 
The measured signals indicate that there are signals coming from the relative time of arrival multipath between received pulses as shown in Figure 6. It can be found from Figure 6 that the first arriving pulse is the first waveform (between points 180 and 249) and the two later arriving waveforms (between points 250 and 299) and (between points 300 and 350) are multipath reflections. Examining the received signal in Figure 6 shows that the shape of a Gaussian signal is derived twice as a modulating sinusoid, which in turn shows the effect of the derivative of transmitting and receiving sensors.

Figure 6 illustrates an example of a bandpass signal at sixty picosecond increments relative to the initial point. For each measurement set and for each sensor, the filter is applied to the bandpass signal, and the motion-filtered signal is brought forth. The points of the motion-filtered signal correspond to possible target distances as can be noted in Figure 6. This clearly shows that the first arriving pulse is the first waveform (between points 180 and 249) and the two later arriving waveforms (between points 250 and 299) and (between points 300 and 350) are multipath reflections.

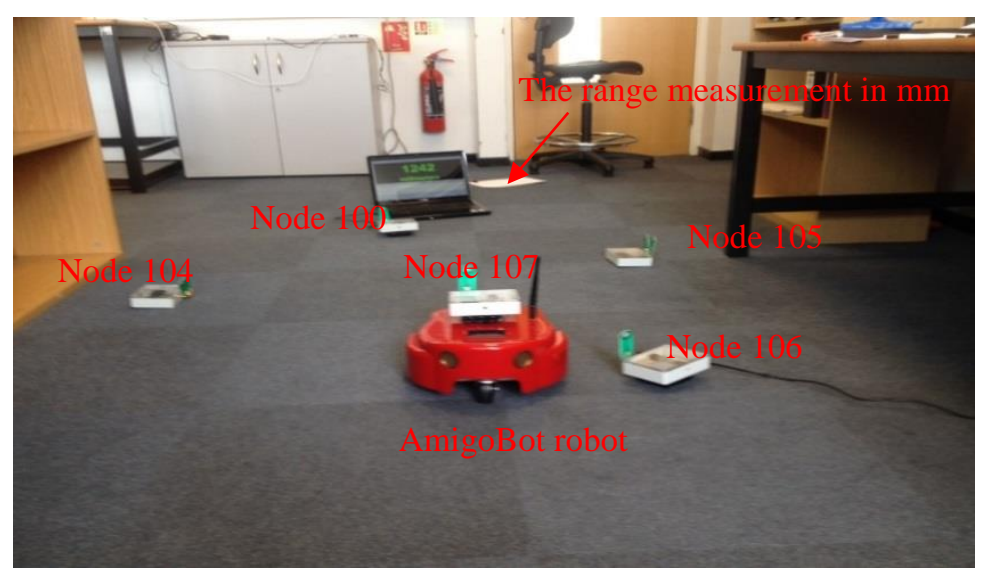

Figure 5: Mechanism of localization with the device descriptions. 


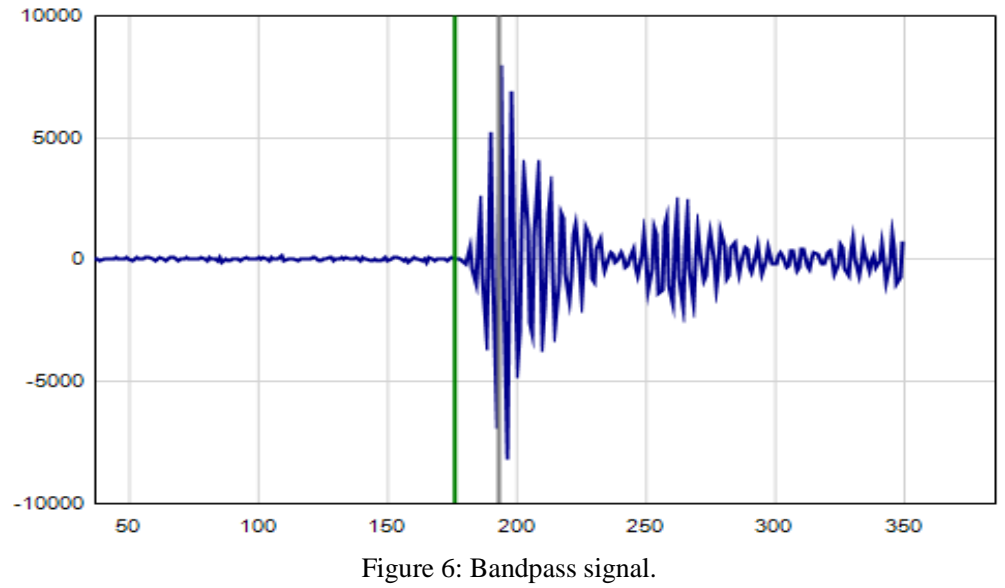

\section{Mechanism of localization}

The mobile robot is localised using the setup and equipment shown in Figure 5. This comprises fives PulsON 410 (four fix nodes and one moving node (robot)), the AmigoBot robot, a laptop and laser rangefinder. The mobile PulsOn 410 is mounted on an AmigoBot robot and four receiver antennas are fixed nodes. Furthermore, the receiving antennas are connected to the receiver wirelessly. In addition, the receiver is connected to the laptop through the Ethernet connection to transfer the received signals for processing.

\section{Experimental results}

The measurements taken were recorded on a laptop and processed. The processed data contain the time stamp, range and velocity information, although the only important information is the fact that these are consecutive measurements with an update rate of every millisecond. Several 2D tests were performed using nodes $100,104,105,106$ as fix nodes and a mobile node 107 (here the node 107 is carried on the back of the Amigobot is a programmable robot) in all experiments. Figure 7 illustrates the initial geometry measurement with just two fix nodes and a mobile positioned at $(0,0)$. The figure shows comparison with the extracted $\mathrm{x}$ and $y$ results from the AmigoBot robot system, so the UWB based localization results can be identified. Figures 8 and 9 show the two signals received by two different nodes (nodes 105 and 106).

For the node 105, the antennas were very close to the transmitter and for the second node 106, the antennas were distant from the transmitter. Figure 9 shows 
that PulsON 410 had high resolution than in Figure 8 and the signals were sampled at sampling period of 1.4073 nanoseconds.

To evaluate the performance of the TDOA estimation algorithm and the localization algorithm, the mobile node 107 was selected and AmigoBot robot containing thousand positions. Ten measurements of the received signal for each position were performed, having thirty TDOA position values. The measurements were conducted in an indoor environment of dimensions $15 \mathrm{~m} \times 8 \mathrm{~m} \times 3 \mathrm{~m}$, with block walls, large glass windows, two doors, tables, concrete floors, ceiling, wooden and metallic furniture. The estimation results achieved in the tests of UWB and AmigoBot robot are shown in Figures 10, 11, 12 and 13. During the experiments, the robot drove from a point $\mathrm{A}$ to point $\mathrm{B}$, and in the last test, the robot drove from $\mathrm{A}$ to $\mathrm{B}$, returned to $\mathrm{A}$, and for safety reasons all experiments were conducted inside the lab. Table 1 shows positions of UWB nodes with corresponding estimated errors.

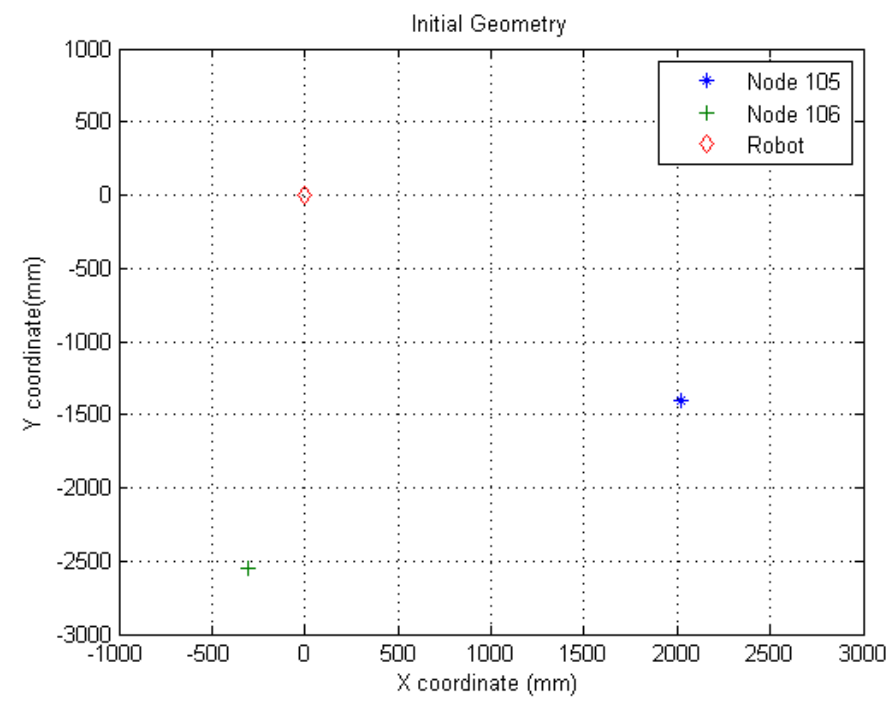

Figure 7: Initial geometry in a testing environment. 

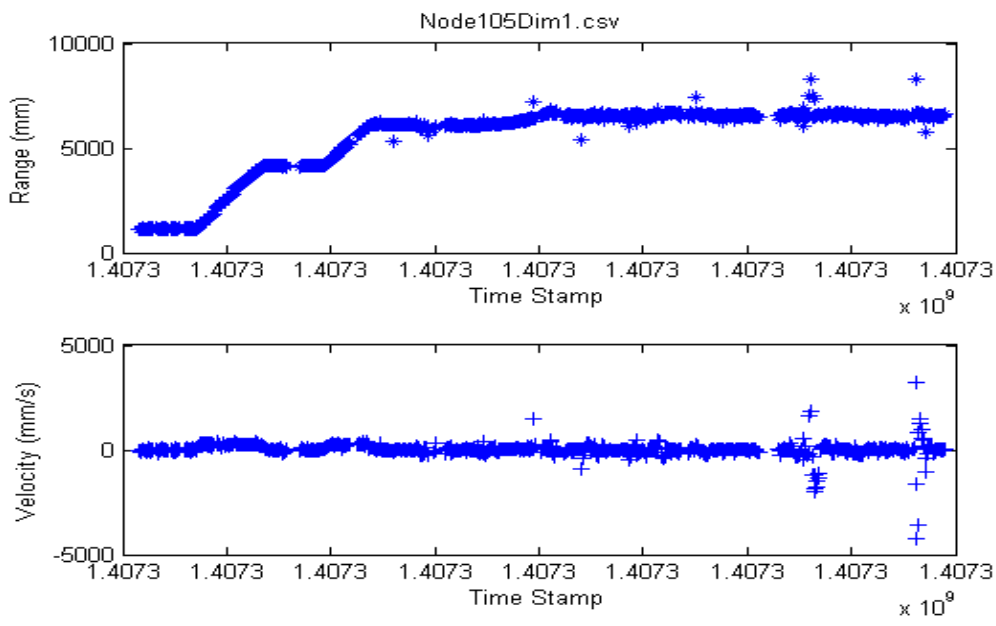

Figure 8: Received signals from node 105 and a bit close to the transmitter.
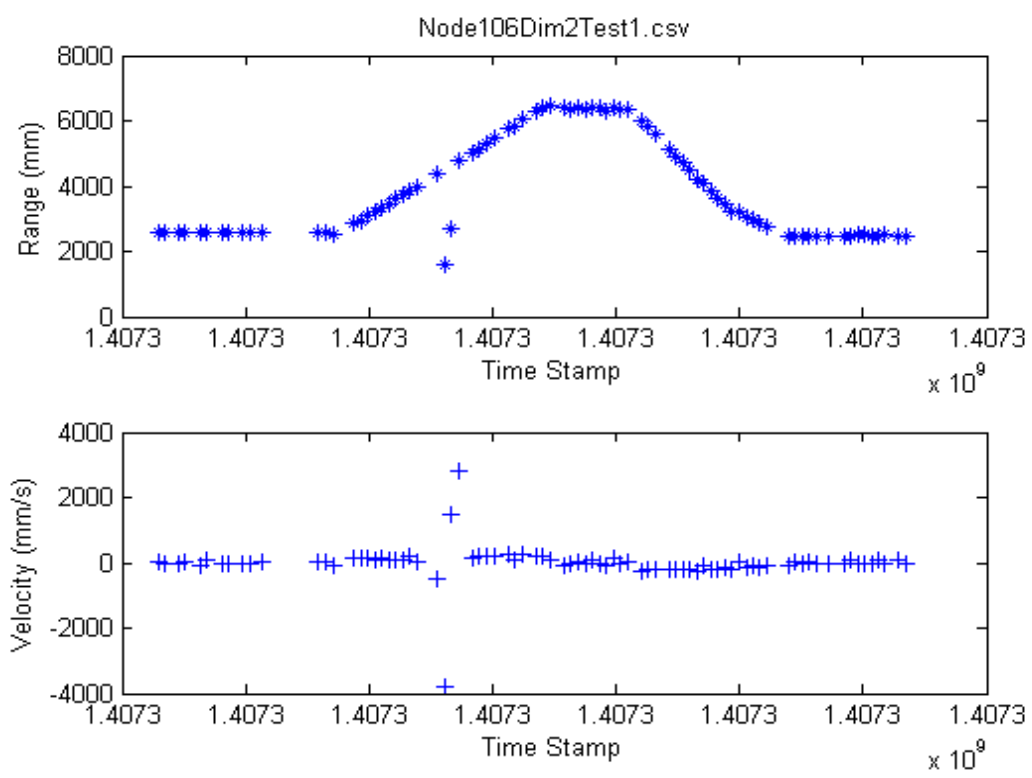

Figure 9: Received signals from node 106 and a bit far from the transmitter. 


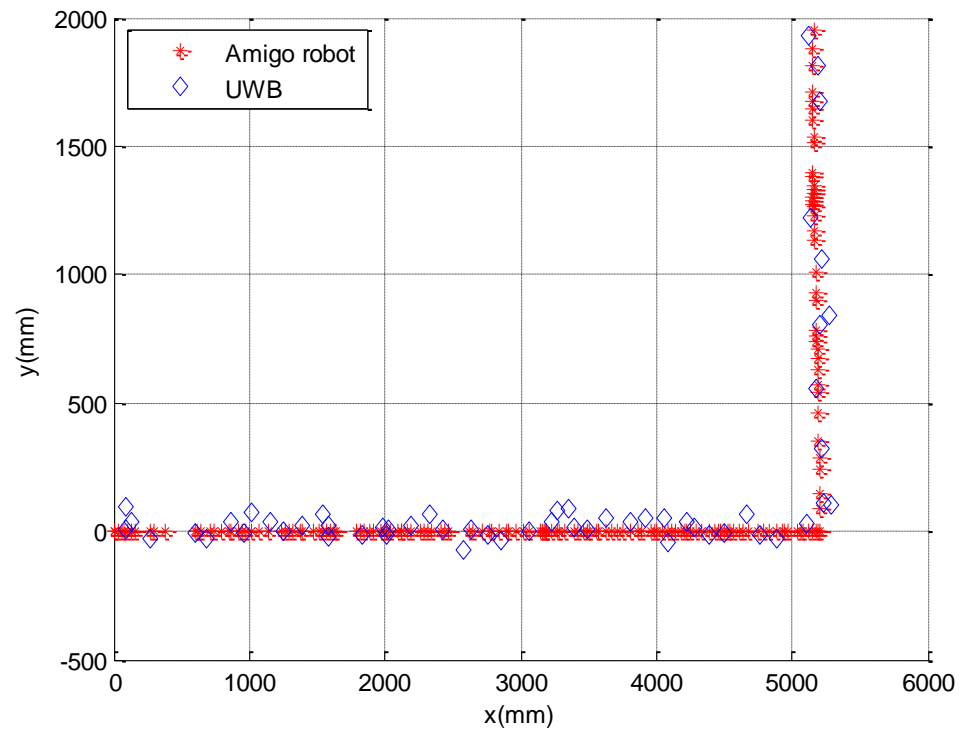

Figure 10: Test 1- positioning of the mobile robot using Odometry \& orientation sensors and UWB from point A to B.

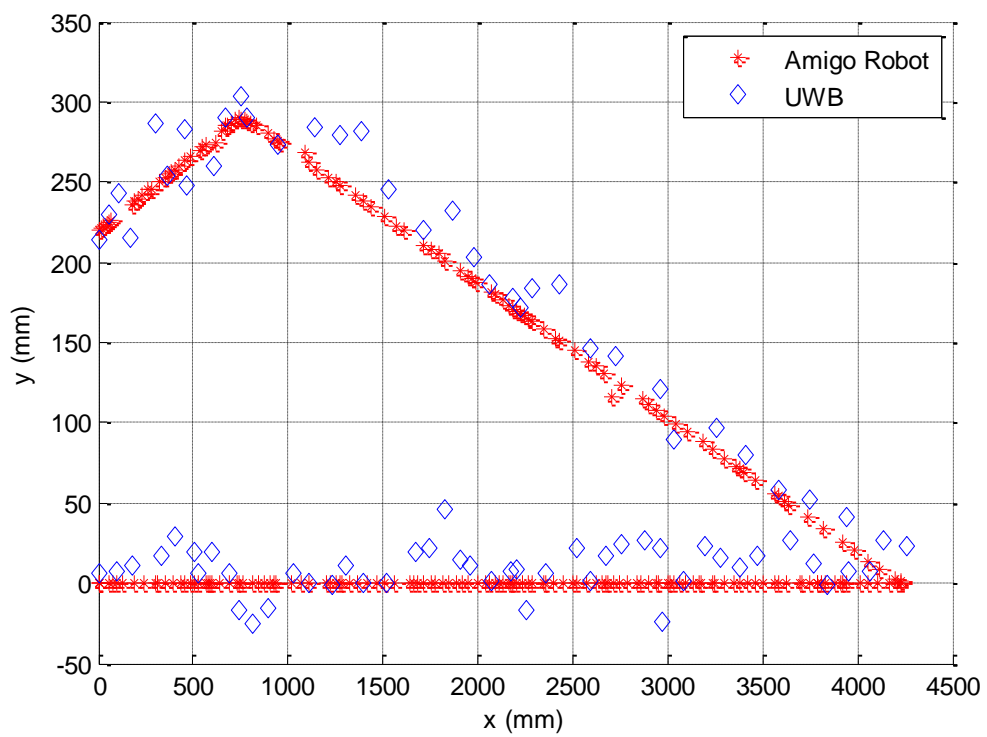

Figure 11: Test 2- positioning of the mobile robot using Odometry \& orientation sensors and UWB from point A to B. 


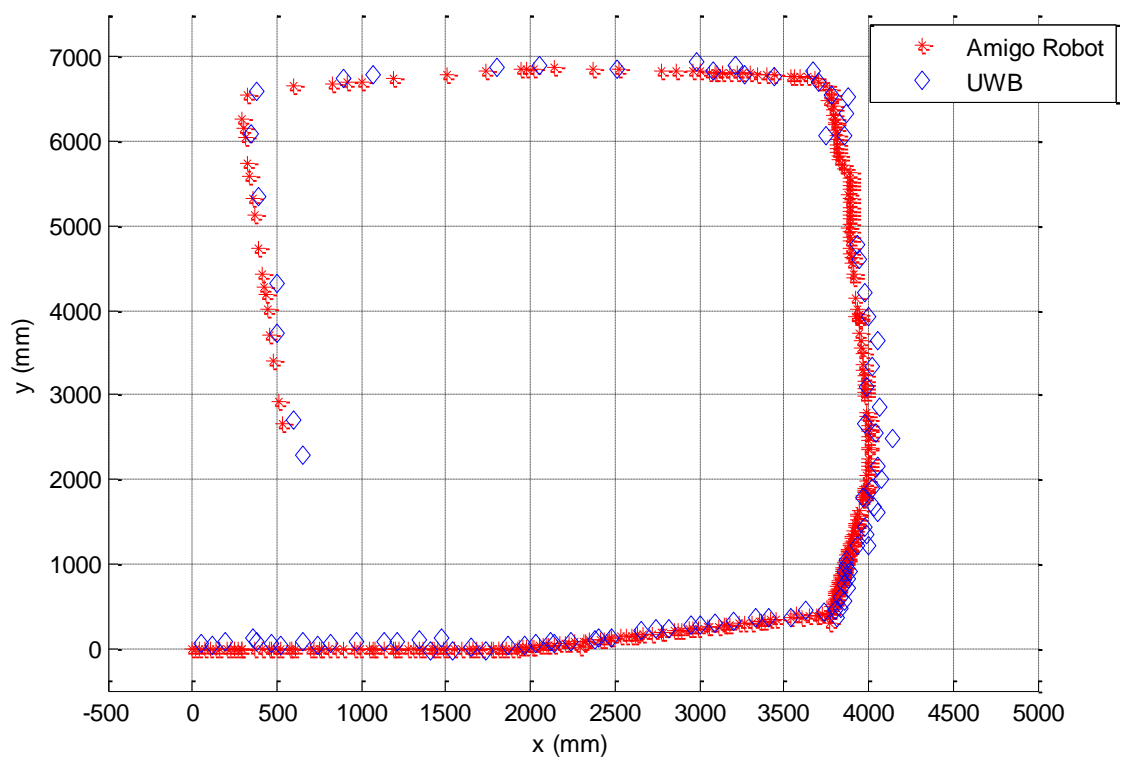

Figure 12: Test 3- positioning of the mobile robot using Odometry \& orientation sensors and UWB from point A to B.

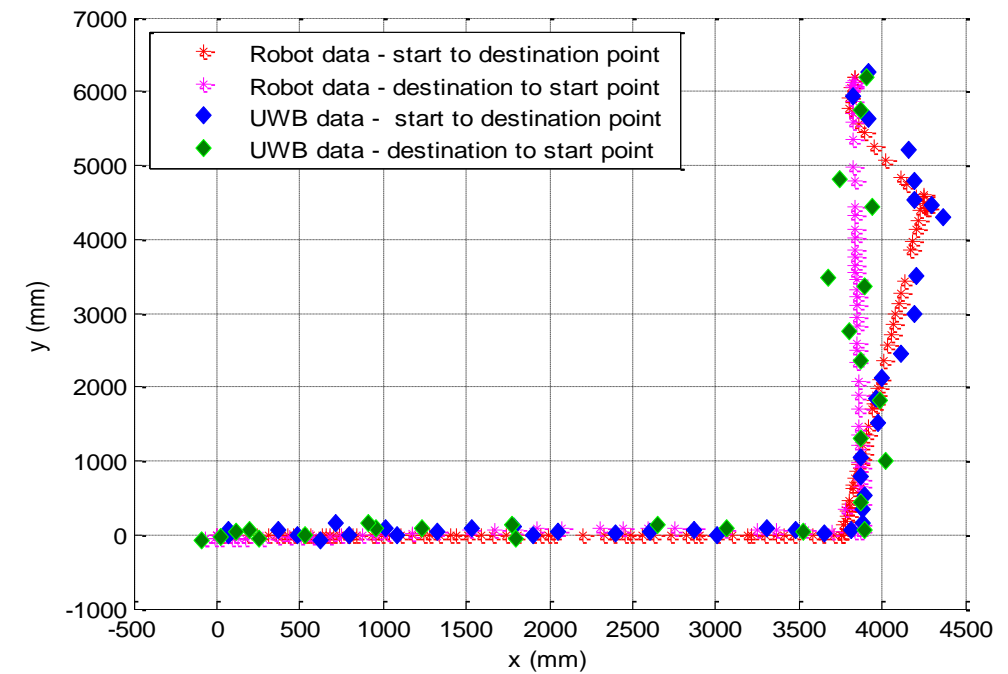

Figure 13: Test 4- positioning of the mobile robot using Odometry \& orientation sensors and UWB from point $A$ to $B$ and from $B$ to $A$. 
Table 1. Position of UWB nodes with the estimated error in mm accuracy.

\begin{tabular}{|c|c|c|}
\hline Connected Node & $\begin{array}{c}\text { Distance from Node 107 in } \\
\mathrm{mm}\end{array}$ & Estimated error in $\mathrm{mm}$ \\
\hline 100 & 4739 & 55 \\
\hline 100 & 8247 & 55 \\
\hline 100 & 3023 & 55 \\
\hline 100 & 1884 & 24 \\
\hline
\end{tabular}

\section{Conclusion}

Experimental investigations have been conducted using UWB signals measured in LOS and NLOS environments for location estimation using the TDOA algorithm. A new measurement scheme has been proposed for improved localization through performance assessment and validation of the use of UWB signals measured in LOS and NLOS indoor environments. The experimental tests have shown that the UWB accuracy is very good with the positioning error between 24 and $55 \mathrm{~mm}$, and the error not exceeding $55 \mathrm{~mm}$. The localization error increases with longer robot travel. Results indicate that a system that uses UWB gives much more accurate position measurement in indoor environments than other localization techniques. UWB provides an accuracy indoors of less than 55 $\mathrm{mm}$ error with a working range of $160 \mathrm{~m}$. The difficulty of obtaining a very accurate positioning system is due to NLOS propagation conditions. The use of UWB TDOA measurement is thus recommended for localisation because of it low cost, low power consumption and low computational complexity and precise positioning capability.

To improve the UWB localization technique in wireless sensor networks in indoor environments, its performance should be evaluated with the objective of proposing new measurement schemes that improve localization and use this information to perform simultaneous localization and mapping (SLAM) to navigate robots to perform cooperative tasks in inspection and non-destructive testing of large safety critical infrastructures. More sophisticated state estimation algorithms such as particle filters using Monte Carlo methods may give more accurate tracking of mobile robots. 


\section{References}

1. M. diBenedetto, T. Kaiser, A. Molisch, I. Oppermann, C. Politano, and D. Porcino, Eds., "UWB Communications Systems: A Comprehensive Overview," Darmstadt, Germany: EURASIP, 2005.

2. M. Guizani, R.C. Qiu, and T. Le-Ngoc, "Ultra-Wideband wireless communications and networks," John Wiley and Sons Ltd, 2006.

3. Z. Sahinoglu, S. Gezici, and I. Guvenc, "Ultra-Wideband Positioning Systems: Theoretical Limits, Ranging Algorithms, and Protocols". Cambridge, Dec 2008.

4. S. Gezici, Z. Tian, G. Giannakis, H. Kobayashi, A. Molisch, H. Poor, and Z. Sahinoglu, "Localization via ultra-wideband radios: a look at positioning aspects for future sensor networks," IEEE Signal Processing Magazine, vol. 22, page 7084, July 2005.

5. S. Gezici, Z. Tian, G. Giannakis, H. Kobayashi, A. Molisch, H. Poor, and Z. Sahinoglu, "Localization via ultra-wideband radios: a look at positioning aspects for future sensor networks," IEEE Signal Processing Magazine, vol. 22, page 7084, July 2005.

6. B. Sobhani, M. Mazzotti, E. Paolini, A. Giorgetti, and M. Chiani, "Effect of state space partitioning on bayesian tracking for UWB radar sensor networks," in IEEE International Conference on Ultra-Wideband, page 120 -125, Sep. 2013.

7. B. Sobhani, E. Paolini, A. Giorgetti, M. Mazzotti, and M. Chiani, "Bayesian tracking in UWB radar sensor networks," in IEEE International Conference on Communications Workshops (ICC), page 47-51, June 2013

8. S. Bartoletti, A. Conti, and A. Giorgetti, "Analysis of UWB radar sensor networks", in IEEE International Conference on Communications (ICC), page 1-6, May 2010.

9. H. Ding, C. Chen, S. Peng, X. Li, and L. Zheng, "Multistatic ultra-wideband localization for NLOS environments", in Second International Conference on Intelligent System Design and Engineering Application (ISDEA), page 380-384, Jan 2012.

10. Activemedia. Amigobot technical manual. Activemedia Robotics, Nov 2000.

11. J. Gwatkin, "Robo-CAMAL:Anchoring in a Cognitive Robot," $\mathrm{PhD}$ thesis in the University of Hull, December 2009. 